\title{
Developing Of Health Promotion Of District Hospitals In Thailand
}

\author{
Jutarat Kumpalanon, Khon Kaen University, Thailand \\ Dusadeee Ayuwat, Khon Kaen University, Thailand \\ Pattara Sanchaisuriya, Khon Kaen University, Thailand
}

\begin{abstract}
The aim of this research is to investigate the health promotion services provided by district hospitals in the northeast of Thailand in order to improve health promotion guideline for district hospitals. The qualitative research was conducted from January 2009 to March 2010. Informants were the managers, the staff members responsible for developing and providing services of health promotion from 19 small, medium and large-sized district hospitals in the Northeastern region of Thailand. The in-depth interview was employed to gather the information analyzed by using content analysis. It was found that the new health promotion trends in district hospitals in the northeast were as follows. The organizational structures for promoting health were obviously defined while active services inside and outside the hospitals were clearly developed which bring health promotion practices to serviced users. Public policy for health was offered in the form of quality control, information management and active services while environmental management emphasized on creating comfort and learning environments for the hospital staff members and serviced users. Collaborations between professional organizations and multi-level networks had been established by district hospitals resulting in services with more accessibility and strengthening local communities. In addition, personal skill development and public health education yielded change to the health behaviors of hospital staff members, serviced users and people in local communities. For health service reorientation, there was emphasis on development of the quality of local community services in forms of professional standard development and assessment of health behavior outcome. The forms of health promotion provided by district hospitals in the northeast that were found were public policy for health promotion, active services, environmental management for health, community strengthening in form of collaboration networks, personal skill development and public health education for changing behavior, and change of health services. In order to improve health promotion, health promotion policies must be clearly defined and collaboration between stakeholders both inside and outside hospitals must be supported.
\end{abstract}

Keywords: Health Promotion; District Hospital; Health Promoting in Hospitals

\section{INTRODUCTION}

$\mathrm{n}$ the age of globalization, the global stream in the name of development continuously expands leading to environmental problems, catastrophes, epidemics, and social and health problems. The increase of people immigration (World Health Organization, 2004) helps in spreading emerging infectious diseases, such as mad cow disease and Ebola virus disease, re-emerging infectious diseases, such as swine flu, and general infectious diseases, such as drug resisting tuberculosis (Department of Diseases Control, Ministry of Public Health, Thailand, 2005). In Thailand, the results of national development have lead to changes in life style of Thai people. While people face more physical and mental problems, as well as, social and moral decay (Ilon, 2003), many people rely mostly on conventional medicine. The health services for people in rural areas provided by district hospitals focus mainly on modern medical treatments. As the population increases, more patients looking for medical treatments overwhelm the hospitals with heavy workloads. In order to prevent health problems, instead of concentrating on medical treatment, many public health organizations have started administering their activities under the policy 
called "Health for All", which mainly focuses on health promotion (World Health Organization, 1986). Therefore, district hospitals have to balance their major responsibilities between medical treatments, communicable disease control and health promotion services (Auamkul, 2002).

Under social changes and the mission toward qualified services, questions are being asked about how the district hospitals can develop qualified health promotion services. Therefore, in this research, the current situation of health promotion provided by the district hospitals in the northeastern region was investigated. Finally, suggestion for improvement of health promotion among the district hospitals in the region was given. The results of this study will be worthwhile in developing health promotion services among district hospitals in Thailand.

\section{METHODOLOGY}

This study was a qualitative research conducted in Public Health Region 14, including the district hospitals located in Nakhonratchasima, Chaiyaphum, Buriram, and Surin Province. The district hospitals involved in this study were both certified and uncertified as the hospitals for health promotion by the Department of Health, Ministry of Public Health in 2008. Regarding hospital sizes, there were 19 hospitals: 6 large (90-150 beds), 8 medium (60 89 beds) and 6 small (10-30 beds) hospitals. The informants were divided into 3 main groups: the group of 19 administrators and staff members appointed by the administrators, the group of 17 staff members from hospital quality improvement sections and the group of 19 staff members from health promoter sections. This study employed in-depth interviews to gather information according to 3 aspects: general information of the district hospitals, the current situation related to health promotion services, and the development of health promotion. Additionally, the authors also employed non-participant observation to gather general information about each hospital and its health promotion services. All activities were conducted between January 2009 and April 2010. Content analysis technique was used to analyze the information presented in the descriptive report.

\section{QUALITATIVE RESULTS}

\section{General Information of the District Hospitals}

Before 2002, according to the state regulation, the organizational structure of every district hospitals had to consist of at least 3 departments: the department of general administration, the department of nursing responsible for providing medical treatment, and the department of health promotion and disease prevention responsible for inhouse clinical services. In the past, the district hospitals weren't responsible for promoting health within their communities. From 1982 - 2001, the district hospitals had increased the number of staff members and budgets and widened their range of services including community services. All district hospitals had actively provided primary health care and pre natal care which focused on protecting patients from illness. From 2002 until now, district hospitals of all sizes have adjusted their organizational structures as clusters, and have focused on administration, providing services and nursing. This has resulted in a budget that has clearly allocated funds for health promotion. After public health reform in 2000, the operation has been actively run, and centers for community health promotion and in-house specialty clinics have been established. More importantly, the cluster for services provided has become involved with the Department of Family and Community Clinical Practice and has distributed more services to the communities. Even the Department of Nursing has had to continuously visit people within the communities and collaborate with the local health stations. This change has lead to the provision of services at the domestic level and to specific groups of patients, and has increased the opportunities and networks to promote health among those groups of people. Moreover, more people have access to the services provided by the district hospitals.

\section{The Situation of Health Promotion Service Provided by the District Hospitals}

In this study, it was found that there were 5 groups of activities created to promote health under the Ottawa Charter (World Health Organization, 1986) including making policies to promote public health, creating supportive environments, strengthening community health care, developing interpersonal skills, and reforming the health service system (Department of Health, Ministry of Public Health, Thailand, 1998) described in detail as follows: 
Making the policy to promote public health. In the past, when the public health policy was created only by the government, health promotion was given priority second to primary health care while services were passively provided and covered limited area of responsibility of each hospital. Later on, Since 1997, the Hospital Accreditation (HA) standard created by the Healthcare Accreditation Institute and the Health Promotion Hospital (HPH) standard founded by the Department of Health have been introduced to hospitals in Thailand in order to improve their quality of service. Mostly, large hospitals have passed HA and HPH criteria while small district hospitals with limited resources rarely pass the criteria. Nevertheless, organizational development (OD) and Excellent Service Behavior (ESB) development are alternative choices for district hospitals to improve their services. In addition, from the in-depth interviews, it was found that the large hospitals usually required participation from all stakeholders to set the health promotion strategic plans. Today, district hospitals have operated under the action plan with key performance indexes indicating the level of success defined by the government while the organizational structures of district hospitals must include the Department of Family and Community Clinical Practice and the Department of Health Promotion.

Creating a supportive environment. It was found that the district hospitals had modified the area around their hospitals to create learning atmospheres and promote the safety of the staff members and service users. In the beginning, the district hospitals had created exercise activities, and provided many items of sports equipment to their staff members. Then the services were offered to other groups, such as the elderly. The environment was used for stimulating health promotion, such as creating health promoting atmosphere in the market and a municipal project called "Comfortable Workplace" where local organizations were urged to promote health by creating exercise activities and sport competitions. In addition, the district hospitals had tested the quality of waste water before releasing it into the environment and operated the waste management systems to reduce negative effects to the surrounding communities.

Strengthening community health care. Besides their primary health care task, the district hospitals needed to encourage health promotion, such as appointing village health volunteers and establishing funding services. The district hospitals also encouraged collaboration from many sectors as networks, such as the network of Public Health Volunteers which focused on chronic diseases and the health care network for laborers. The medium-sized hospitals linked their networks to local artists allowing easier and more understandable communication to local patients by using story telling. The monks in the community also played a role in creating better mental health, taking care of terminally ill patients, and organizing camps to promote the campaign called "No Alcohol in a Temple." Furthermore, after the success of the network of Public Health Volunteers, several networks have been established such as the network of elders and the group of AIDS patients.

Development of personal skills for health promotion. Skills for health promotion cover from the behavior of each individual to behaviors of groups to take care of their health. In the community hospital, the development of personal skills for health promotion was mainly determined by the health policy of the hospital. For example, there was an annual health check-up in order to assess the risk that personnel face from workplace hazards. Several healthcare courses such as self care, holistic care and especially primary care had been taught in small-sized hospitals. There were also forums for learning and sharing knowledge about healthcare and behavior changing. Some medium-sized hospitals motivated people to think about their health by inviting experts to come to help.

Several activities for promoting the patients' and relatives' behavior such as creating health awareness for target groups were focused. There were learning processes targeting patients and relatives to educate them how to care for themselves before going home, for example, a camp for adjusting behavior. In the medium-sized hospitals, there were some caring innovations for patient's relatives such as a program for diabetic and high blood pressure patient care which was given during a learning period of patient's relatives and a program for infectious patient care. These programs provided a pre-counseling section helping the users to realize their own potential and see the benefit of participating in different activities. For adjusting the health behaviors of people in the community, there were communicative activities to promote health awareness from the hospital to the community by creating health communities such as providing camps for training community health care and visiting activities operated by coordination between the hospitals and patients in the community. 
Reforming the health service system. The present health service system had been changed to become a holistic care process where health problems were analyzed using local information as well as health information from the hospital database. The communicative medical units in local communities had been set up in order to gather information in the communities. The service tasks had been separated between the outpatient clinic, the extended clinic and clinical specialist. In addition, in the small-sized hospitals, it was found that nurses had played a key role in providing continuity of care assuring that patients, families and relatives can take care of their health problems. The continuity of care could go hand in hand with nursing standard quality assurance (QA) while the district hospitals provided more health promotion in the community. In addition, there were processes connected to the community to promote the health awareness both in the hospitals and in the community. However, these processes lacked participating activity and continuity.

In summary, the present health promotion of the district hospitals have changed from the old concept of health promotion based on the medical treatment model to a new concept of health promotion. It creates the process of health promotion, and also brings about the public health policy of the organization. The organizations make the objective plan, create the environment, and change the hospital system. This leads to community participation and strengthens the development of health network in the community.

\section{HEALTH PROMOTION IN THE COMMUNITY HOSPITALS}

The analyzed data was obtained from in-depth interviews with the boards of district hospitals, the responsible health groups of community hospitals, and the responsible health promotion groups of community hospitals (Department of Health, Ministry of Public Health, Thailand, 2005). The results according to the new concept of health promotion and the health promotion services were found as follows:

Public policy for health promotion. It was found that, previously, before 2000, the hospitals had set a policy for directing organization according to mission and vision established by the process of personal participation which did not focus on health promotion. The hospitals had found that the cost of medical treatments were more expensive than the cost of health problem prevention. Therefore, later on, as prevention of health problems have become of interest and due to limited budget, the community hospitals had changed direction toward health promotion. The hospitals had created clear health promotion policies. There were key performance indicators for health promotion assessment defined by the government and policies linked to hospital accreditation and health care service. Currently, under the health promotion policy, the community hospital has employed modern organizational analysis tools such as Strength-Weakness-Opportunity-Treat (SWOT) analysis and Balance Score Card in the process of strategic planning for health promotion which resemble the paper by Ziglio, Hagard, and Griffiths (2000).

For the roles and responsibilities of the community hospital staff, originally, the Department of Health Promotion and the Department of Health Sanitation and Prevention Diseases were in charge of the health promotion. At the present time, the Department of Health Promotion has been changed to the Department of Family and Community Medicine where the scope of the department job includes not only health promotion of serviced users but also those of families and communities. Also, there are committees responsible for developing the quality of service and health promotion of the hospitals. The some study indicated that the success of health promotion depended on the organizational structures and committees of the hospitals (Yeatman \& Nove, 2002).

For health promotion activities, previously, the activities were separated from other activities and only the Department of Health Promotion had taken responsibility for health promotion. Now, the community hospitals have integrated the health promotion into every hospital department, brought some activities to communities and created campaigns to encourage all parties to join health promotion activities. The hospital boards had fully supported the activities promoting both physical and mental health of hospital staff who were considered as role models in health promotion for people. Some activities such as patients' house visits could improve the quality of service and health care and can cause organization adjustment.

In summary, regards to the development of health promotion in the community hospitals, public policy in health promotion, the potential benefits, and the limitation of the organization, a clear concept of health promotion has been developed. The health promotion policy, along with the quality of service policy, has been connected to the strategic plans that link the hospitals to the communities. 
Proactive services provided. The proactive services are the services that consist of health promotion and disease prevention along with medical treatments. It was found that the hospitals had provided proactive services by having health promotion clinics, patient house visits by medical professional teams and the health promotion networks. However, all levels of the district hospitals had shown fewer proactive services for health promotion than for medical treatment. In order to achieve hospital accreditation, the community hospitals took a holistic approach, namely proactive services, to health care by using quality control and health promotion policies. This was consistent with the developing process of health promotion in quality development of the hospitals (Whitehead, 2004). However, proactive services of some community hospitals, especially the small-sized hospitals had emphasized on Organization Development and Excellent Service Behavior instead of services related to hospital accreditation due to their limited budgets. In order to obtain overall information, some district hospitals had collected information from all departments and patients' house visits and used this data for implementing the strategic plan. Some medium and small-sized hospitals had established data centers connected to local health clinics via telemedicine networks. Nowadays, all sized hospitals and some clinics have shared patient data recorded in data centers between each other. According to the study, utilizing the community information resulted in better health promotion performance (Yannis, Elpida, Kyriaki, Nikolaos, \& Dimitra, 2004). In summary, proactive services had help increase collaboration between all parties and improve quality of service of the hospitals.

Environmental management for health. In the past, in both hospitals that had been assessed for health promotion hospital accreditation and those that had not been assessed, environmental management for health had been taking care of physical structure such as caring of hospital landscape, focusing on cleanliness and beauty, building a health park, having work-out activities, and maintaining fitness equipment. Nowadays, many hospitals have now changed their landscape to become learning resources. Hospitals of all sizes have developed physical structures where the surroundings could help in the process of health promotion. This strategic plan in developing physical structures has been found to be efficient and supports the activities in those areas (Ziglio et al., 2000).

For physical management, formerly, physical management was meant to be only for the purpose of convenience and security. For example, there were some warning signs in some safety precaution zones such as handicap ramps showing instruction guidelines and some direction signs to inform people where the places such as restrooms were. Later, direction of physical management has changed toward creation of learning resources and security improvement. It was found that, in the small hospitals that had not passed HPH accreditation as well as in the municipalities, there were community projects that focused on creating attractive landscapes making surrounding environments more pleasant. Regarding environmental management in the society, there were systematic waste management systems, for examples, waste sorting for recycling and wastewater treatment systems. Nowadays, in every hospital, water quality has been monitored regularly while waste management systems have been implemented at public health clinics. The hospitals also encouraged the municipalities to have an exercise programs for the elderly by creating health promotion environments and letting the municipalities take care of the activities on their own. This could help support the health of the local communities by creating healthy work place activities.

In summary, the development of health promotion in the district hospitals includes environmental management by creating pleasant landscape and learning environments in order to promoting health. The landscape is adjusted not only to prevent any dangerous situations that might happen in all service units but also to accommodate an environment for the promotion of good health.

Strengthening healthcare in communities. In the past, there were only health activities created by public health volunteers of the villages. Later, as health promotion became more popular, there were some support from other health associations in the form of health promoting activities created by the public health volunteers and other volunteers, for example, groups called "Friends Help Friends" (Phuan Chuay Phuan), groups of laborers in the factories, and groups of community leaders. These groups were extended from local organizations to the communities in order to increase the potential of the primary care units and to strengthen the community networks. The study found that health promotion worked well when teamwork was developed (Yannis et al., 2004). Such teamwork focused on promoting health in the community through primary care units and nursing teams belonging to health networks. There were also other health associations such as groups of monks, heartfelt volunteers, local leaders, and community leaders to help promote the personnel caring behaviors among their groups. Such activities were also found in the district hospitals that had not passed the assessment of health promoting hospitals 
accreditation. Strengthening healthcare in communities found in the study was consistent with the self-health care promotion and the participating process (Bhuyan, 2004).

In summary, in terms of strengthening the communities, it was found that, in order to develop health promotion in the district hospitals, establishment of self-healthcare groups could increase the potential of the primary care units and community networking. The community networking and the self-care process had extended to other groups which was consistent with the strategic plan of continuous health promotion which promoted healthcare within the community (Ziglio et al., 2000).

Knowledge and personal skills development. The development of knowledge and personal skills of the staff was initially the result of personnel training in the hospitals called Organization Development. Later, the development of personal skills in health promoting has been integrated to connect with other related organizations, departments and health networks. Nowadays there are learning and sharing forums for health promotion and quality of service improvement, expert corners, innovative oriented follow-up treatment processes by visiting patients' houses in the communities. However, in the small district hospitals, which have not passed the health promoting assessment, personal skill development was not obvious and not often.

For the health behavior adjusting activities in the community, there were follow-up activities, camping activities to help the patients, relatives, friends, and the caretakers of the patients to make behavioral adjustments as well as public hearings about health policies and public communication in order to promote health advancement. In some cases, the patients were taught several courses for checking health problems and how to take care of themselves at home (Detmer, 2003) before sending the patients back home. For example, in the diabetic mentor activity, patients had shared knowledge, obtained from their own experiences, about how to take care of themselves.

According to the survey, the knowledge and personal skills development indicated that the district hospitals which had a solid strategic plan for personal skills development among their hospital staffs had analyzed the health potential of their staffs, developed the skills of self-care and behavioral adjustment for their patients and their patient's relatives after returning home, and had community participation which could extend the awareness of health promotion into the community.

In summary, in this aspect, health promotion is a process in which the patients can learn how to take care of themselves through community participation and the development of the organization that demonstrates to patients and relatives ways to adjust their behaviors after going back to their homes. Caring for themselves after going back home was well-being behavior that results from the health promoting process (Bhuyan, 2004; Galloway, 2003; Whitelaw, Martin, Kerr, \& Wimbush, 2006 ). Community participation can help by extending the self-care process among the community.

Health services reorientation. The surveyed results indicated that, starting from sharing patient data via staff networks, the district hospitals had expanded their sharing data networks to the community medical centers which were separated from the outpatient clinics. While the nursing teams who made visits to patients' houses were responsible for collecting the data. Nowadays, the primary holistic care and nursing teams have adjusted their roles toward connecting local organizations and also having continuous treatment for patients in all areas. Some studies found that modifying the overall hospital service system was one of the significant factors to improve the hospital's performance (Yeatman \& Nove, 2002; Whitelaw et al., 2006). The modifications covered the development of information systems and communication systems in order to improve health promotion (Haynes \& Cook, 2007). However, one problem in health service reorientation found was that some district hospitals had a lack of potential staff members who could run community medical centers and community health centers.

To extend the health services including supervision in respect to laws, morality, and human rights, to people, families, communities, and health centers, there were some adjustments needed of the roles of nursing to be consistent with the professional nursing standard. For instance, the care plan had been made before patients returning home, the cultural adjustment in the workplace and the strategic plan had been changed and the holistic analysis were connected to the local organizations. There were leaders of the health promotion, public hearings, research, and the extension of special clinical services and clinical specialists. All disabled patients and 
disadvantaged patients were treated without discrimination. Nevertheless, in some small district hospitals which had not passed the health promoting hospital accreditation, services for disadvantaged people were not obvious. In addition, according to the health promotion assessment, initially, only health activities were evaluated. Nowadays, all processes and factors related to health promotion including health promotion activities, ability of patients to care for themselves, health service accessibility, and community strength are evaluated.

Therefore, in respect to the adjustment of the health service system, the health promotion development in the district hospitals had shown that there had been some adjustments in primary health care with regards to holistic care and nursing teams. Moreover, the change of hospital staff had been adjusted along with the professional standards and connections to local organizations. There was also the continuous change of medical service for the patients in all areas.

In summary, several factors supporting the development of health promotion in the district hospitals were found including public policies for health promotion, proactive service, environmental management, strengthening healthcare in communities, knowledge and personal skills development, and health service reorientation. First, health promotion policies had been of interest. The district hospitals had changed their organizational structures to serve their strategic plans in health promotion. Proactive services, community health centers, special clinics, activities linking to communities were implemented as tools for holistic health care. For the provision of proactive services, such as increasing the degree of performance according to the strategic plan, the district hospitals used the hospital accreditation and health promoting hospitals standard to improve all hospital units. Teamwork and community networking also played an important role in boosting quality of service of the hospitals. Information systems were implemented and utilized throughout community networks. Regarding environmental management for health, the landscape had been rearranged not only for improving security but also to create a learning atmosphere and promote health. Self-care groups, groups responsible for primary care, health networks within the community, and extended community groups had been established for strengthening the communities. There also were training courses for staffs to develop knowledge and personal skills in health promotion. The skills development and behavioral adjustments for patients and relatives before returning home and the participation in self-care and extended community had been established. Moreover, regarding the health service re-orientation, a change has been made from the primary care to become holistic care with professional nursing teams. Also, the duties of service centers and staff have been continuously changed in order to be more consistent with professional standards and to be connected to local organizations.

\section{CONCLUSION AND SUGGESTION}

\section{Conclusion}

The study of the development of health promotion in the district hospitals in Thailand consisted of the district hospitals overall information, the services of health promotion in the district hospitals, and the development of health promotion in the district hospitals. In general, the district hospitals have defined their structures to suit their mission. Each hospital clearly has its own administration, services, and treatments. It has been found that after the reformation of the healthcare system, the hospital operations have become more proactive service oriented. This can be seen in both health community centers and in clinical specialists. It can be especially seen in the mission of administration, in which there is a clinical family and community practice that tends to promote and take a greater part in health promotion. Even the mission of the nursing groups is consistent with that of the health center when they visit the patients' houses and continuously give care to the patients. These actions lead to the concept utilized at the family level and in the patient groups. There are numbers of health networks within the community which result from the participation of health promotion in the hospitals and communities.

The condition of health promoting service is used mainly to focus on primary health care by having a health policy from the public only. The performing service is in the form of prevention and has specific areas of responsibility. The environmental management for health promotion did not realize its effect on the community because they did not have knowledge and understanding of environmental management. For example, there were both improper waste disposal and improper wastewater treatment. Therefore, the hospitals have started the process and have integrated the health promotion into different units. There has also been health networking which has been 
the driving force for health promotion in both communities and hospitals. Nowadays, the development of health promotion in the district hospitals is a new concept that focuses on holistic health care.

The priority of health promoting development is knowledge and personal skills development of the users (patients). The training for new behaviors of patients and relatives needs to be done before patients can be sent home, so they have to have knowledge to take care of themselves. For example, in the diabetic mentor activity, the patients get to practice how to take care of themselves after going back home and continuously caring for themselves. The objective is to create the knowledge and to develop the personal health skills such as behaviors for the patients in the community. People in the community acknowledge the hospital policy. There are communicative activities on health promotion. These activities can extend further into the communities, and the hospitals hope that they can respond to the needs of the communities and the users.

Regarding the development of health promotion in the district hospitals, it has been found that all district hospitals have set their health promoting policy. However, they do not have clear guidelines about how to bring this policy to the community. The policy should let the people in the community participate in making health policies. There are various participating activities, but they do not explain how each different group could participate in each activity. In conclusion, there should be clarity about the different aspects; especially arranging the activities either inside or outside the hospitals, developing a health network, and understanding the roles of each health network.

\section{Suggestion}

From this research, recommendations that could help promoting health promotion in district hospitals in Thailand are given below:

1) In order to understand what people really need, the study of health promotion development should include the case studies of the serviced users (patients) who receive the health promotion services from the district hospitals.

2) The policy in health promotion should be clear.

3) The setting and planning of the participation in the district hospitals should be more visible and comprehensible. This research found that there were various activities, but only hospital staffs, people in the communities, or health promotion volunteers were usually in charge of the activities but there was no participation from other organizations.

4) The health promoting activities should be reviewed after they are completed in order to improve them and make them better.

5) The development of the health promoting networks, such as the health promotion volunteers and other volunteer groups, should have visible roles in order to make health promotion more effective.

\section{AUTHOR INFORMATION}

Jutarat Kumpalanon ${ }^{\dagger}$ is currently a PhD student in the development science program, Faculty of Humanities and Social Sciences, Khon Kaen University, Khon Kaen, Thailand. She received a Master Degree from Srinakharinwirot University, Bangkok, Thailand in 1993. Her current research focuses are on Health Promotion. Email: tarat_kun@yahoo.com. Corresponding author.

Dusadee Ayuwat is corresponding author, currently working at the faculty of Humanities and Social Sciences, Khon Kaen University, Khon Kaen, Thailand. She finished her doctoral degree in Demography from Institute for Population and Social Research (IPSR), Mahidol University Bangkok, Thailand in 1997. Most of her research focuses on demographic issues and organization capability such as migration and well-being, international labor, human security and community development capability. Email: dusayu@kku.ac.th

Pattara Sanchaisuriya is currently working at the department of nutrition, the faculty of Public Health, Khon Kaen University, Khon Kaen, Thailand. Email: pattara@kku.ac.th 


\section{REFERENCES}

1. Auamkul, N. (2002). Health Promoting Hospital from concept to practice. Retrieved from: http://advisor.anamai.moph.go.th/hph/concept.html.

2. Bhuyan, K. K. (2004). Health promotion through self-care and community participation: Element of a propose programme in the developing countries. BMC Public Health, p.4-11.

3. Department of Diseases Control, Ministry of Public Health, Thailand. (2005). Emerging-re-emerging infection disease. Retrieved from: http://beid.ddc.moph.go.th/th/index2.php?option=com.conten\&task=view.

4. Department of Health, Ministry of Public Health, Thailand. (1998). Promoting Health Promotion. Retrieved from http//advisor.anamai.moph. go.th /hph/branch/center.html.

5. Department of Health, Ministry of Public Health, Thailand. (2005). Development Hospital Accreditation to Health Promoting Hospital HPHA. Retrieved from: http//advisor.anamai.moph.go.th/hph/hphosp/ghphbz06.html.

6. Detmer, D. E. (2003). Building the national health information infrastructure for personal health, health care services, public health, and research. BMC Medical Informatics and Decision Making, 3(1), 1-12.

7. Haynes, C. \& Cook, G. (2007). Greater Manchester Health Promotion in Hospital Audit in association with GMEC. Summary Greater Manchester Health Promotion in Hospital Audit report 2007, 1-10.

8. Ilona, K. (2003). The Contribution of the World Health Organization to a New Public Health and Health Promotion. American Journal of Public Health, 93(3), 383-388.

9. Galloway, R. D. (2003). Health Promotion: Causes, Beliefs, and Measurements. Clinical Medicine \& Research, 1(3), 249-258.

10. Whitehead, D. (2004). The European Health Promoting Hospital project: how far on? Health Promotion International, 19(2), 5-14.

11. Whitelaw S., Martin C., Kerr A., \& Wimbush E. (2006). An evaluation of Health Promoting Health Service Framework: the implementation of setting based approach with the NHS in Scotland. Health Promoting International, 21(2), 235-288.

12. World Health Organization. (1986). The Ottawa Charter for Health Promotion First International Conference on Health Promotion. Ottawa.

13. World Health Organization. (2004). Standards for Health Promotion in Hospital. WHO European office for Integrated Health care services. Retrieved from: http://www.euro.who.int/ data /assets/pdf file 10006/99762/ e82490.pdf

14. Yannis T., Elpida P., Kyriaki T., Nikolaos A., \& Dimitra T. (2004). Evaluation of the participation of Aretaieion Hospital, Greece in the WHO Pilot Project of Health Promoting Hospitals. Health Promotion International, 19(4), 45-68.

15. Yeatman, H. R. \& Nove, T. (2002). Reorienting health services with capacity building: a case study of the Core Skills in Health Promotion Project. Health Promotion International, 17(4), 341-350.

16. Ziglio E., Hagard, S. \& Griffiths, J. (2000). Health promotion development in Europe: achievements and challenges. Health Promotion International, 15(2), 210-265. 


\section{NOTES}

